\title{
Action conjuguée des Hétéroptères Miridae et du déficit hydrique dans la malformation des graines de luzerne
}

\author{
René BOURNOVILLE $(*)$, Bernard BOURDONCLE $\left(^{*}\right)$ \& Kyran DONTCHEV $\left({ }^{* *}\right)$ \\ avec la collaboration technique de Pierre CANTOT $\left(^{*}\right) \&$ Patrick DECOURT (*)
}

(*) I.N.R.A., Laboratoire de Zoologie, F 86600 Lusignan.

(**) Institut des Fourrages, 5800 Pleven, Bulgarie.

RÉSUMÉ

Luzerne,

Etat des graines,

Adelphocoris lineolatus,

Lygus rugulipennis,

Etude au champ.

Humidité du sol.
On a étudié, dans les conditions de la pratique agricole et dans des parcelles expérimentales, l'action des punaises Miridae et du déficit hydrique sur la malformation des graines de luzerne. Dans le premier cas, l'analyse de l'humidité du sol et l'appréciation des niveaux de populations de Lygus rugulipennis Popp. et d'Adelphocoris lineolatus Gocze permettent d'établir, en année pluvieuse, une corrélation négative et significative entre les malformations des graines et les effectifs de punaises. Cette relation n'existe plus en année sèche, lorsque les déficits hydriques masquent le rôle des insectes. Dans les parcelles expérimentales, on a réalisé des infestations artificielles de punaises sous cage dans des parcelles irriguées ou non irriguées. Les punaises augmentent, dans la majorité des cas, les pourcentages de graines malformées. Les autres types d'action des punaises, notamment leur influence dans la chute des gousses, n'étaient pas quantifiés dans cette expérience.

\section{SUMMARY}

Lucerne,

Condition of seeds, Adelphocoris lineolatus, Lygus rugulipennis, Field study,

Soil humidity.

\section{Combined action of mirid bugs and water shortage on seed malformation in lucerne}

We have studied the joint action of soil humidity and abundance of bugs (Lygus rugulipennis Popp. and Adelphocoris lineolatus Goeze) on the state of the seeds of lucerne inside the pods. The investigation was first made under field conditions by sampling population of bugs and measuring soil humidity. Under rainy weather conditions, we obtained a significant correlation between shrivelled seeds and populations of bugs. In another experiment we tested the action of the bugs in experimental plots in seed-crops of lucerne, with or without irrigation. With artificial infestation of bugs, under dry or irrigated conditions we demonstrated an increase in mirid-damage to the seeds. Other types of action of bugs on the plant (drying and dropping of flowers and pods) were not investigated in our study.

\section{INTRODUCTION}

Les Hétéroptères Lygus rugulipennis Popp. et Adelphocoris lineolatus Goeze (Miridae) occasionnent à la production de semences de luzerne des dégâts variés (dessèchements des bourgeons florifères, chute des fleurs et des jeunes gousses, malformation des graines dans la gousse) que nous avons définis lors d'études en conditions contrôlées (BOURNOVILLE, 1975). En raison des multiples causes de « coulure physiologique » des organes floraux et fructifères de la luzerne et du potentiel floral très important de cette plante, l'observation de l'état des graines dans les gousses mûres est une évaluation quantifiable des dégâts des Miridae dans les conditions de la pratique agricole, même si elle n'est que partielle (ROTREKL, 1973). Cependant, des études agronomiques (PEYREMORTE, 1974) ont également prouvé que des déficits hydriques en phase de maturation des gousses entrainent également des réductions du poids des graines. Ces altérations de la semence, qu'elles soient occasionnées par les insectes ou par le manque d'eau, se traduisent par des symptômes variés allant de la graine légèrement déformée à un dessèchement presque complet. Nous avons précédemment prouvé que la faculté germinative de ces graines diminue très rapidement lorsque le poids de 100 graines est inférieur à $0,17 \mathrm{~g}$ (BOURNOVILLE, 1976). Par ailleurs, l'observation de ces dégâts impose que l'on prélève, avant la récolte, des gousses au champ car les graines malformées sont éliminées au battage. On ne les retrouve pas non plus dans les appareils de micro-battage de laboratoire.

Afin de saisir la part du déficit hydrique dans la dépréciation de la qualité de la graine et d'estimer les dégâts des seuls Miridae en conditions extérieures, nous avons entrepris de 1974 à 1977 diverses expériences, d'une part, en pratiquant des infestations artificielles de punaises dans des parcelles d'essais irriguées et non irriguées et, d'autre part, en estimant les effectifs de ces insectes présents dans des champs d'enquête dont on a par ailleurs estimé l'état des réserves en eau des sols.

\section{MATÉRIEL ET MÉTHODES}

\section{Les champs d'enquête}

12 parcelles de luzerne porte-graines ont fait l'objet, en 1974 et 1975, d'une étude sur les facteurs de rendement (Bournoville \& Bourdoncle, 1978). Ces champs 
sont situés dans les zones de Lavaur et de Gaillac (Tarn). Pour ce qui intéresse la présente note, nous avons défini l'abondance des Hétéroptères Miridae par des prélèvements réguliers d'insectes tous les $10 \mathrm{j}$ en 25 coups de filet fauchoir. Ces effectifs sont exprimés sous forme d'une note d'abondance variant de 1 à 5 , tel que nous l'avons exposé précédemment (BouRNOVILLE \& Delaude, 1975). Le tableau 1 rappelle le système de notation. L'état des graines dans les gousses est apprécié par l'ouverture de 100 à 200 gousses selon le pourcentage de graines qui sont malformées (BouRNOVILLE, 1976).

\section{TABLEAU 1}

Notes d'abondance établies selon les effectifs de Miridae prélevés en 25 coups de filet (larves et adultes d'A. lineolatus et L. rugulipennis).

Abundance scores of Miridae according to 25 sweep-net samples.

\begin{tabular}{cccccc}
\hline \hline Notes & 1 & 2 & 3 & 4 & 5 \\
\hline $\begin{array}{c}\text { Effectifs } \\
\text { Numbers }\end{array}$ & $<25$ & $25-50$ & $50-75$ & $75-125$ & $>125$ \\
\hline \hline
\end{tabular}

Les gousses sont tirées au hasard dans des lots d'inflorescences récoltées sur 25 tiges principales, tous les 2 niveaux de fructification, afin de représenter au mieux l'étalement de la floraison dans l'espace et dans le temps. Les déficits hydriques ont été estimés par des analyses de l'humidité du sol. A trois dates différentes, correspondant au bourgeonnement, à la floraison et à la fructification de la luzerne, on fait des prélèvements de sol, à l'aide d'une tarière à moteur. Des échantillons sont réalisés tous les $20 \mathrm{~cm}$ jusqu'à $1,20 \mathrm{~m}$ de profondeur. L'humidité des divers niveaux est mesurée au laboratoire. Lors de la $1^{\text {re }}$ série de mesure, les valeurs caractéristiques des sols des divers champs ont été estimées: humidité à la capacité au champ et humidité au point de flétrissement temporaire. C'est lorsque la plante a subi un déficit de l'ordre de 3 points d'humidité (seuil variable selon la nature du sol) par rapport au point de flétrissement temporaire (estimé arbitrairement par les $2 / 3$ de la capacité au champ) que l'on peut estimer qu'un déficit hydrique se manifeste.

\section{Les infestations artificielles}

Elles ont été réalisées dans 2 parcelles expérimentales, distantes de quelques centaines de mètres, du domaine du Centre de Lusignan, en 1976 et 1977 . Ces champs ont été semés en sol nu en avril 1976. L'une des 2 parcelles était irriguée. Le contrôle de l'alimentation hydrique a été assuré au moyen d'une sonde à neutrons par des mesures d'humidité du sol tous les $10 \mathrm{~cm}$, jusqu'à 1,60 m de profondeur. $\mathrm{Au}$ début du mois de juillet, on installe, dans chaque parcelle, 5 cages cylindriques de $50 \mathrm{~cm}$ de diamètre et $2 \mathrm{~m}$ de hauteur dont les parois sont constituées de nylon à maille très fine soutenu par une armature métallique. Un traitement insecticide avec de l'endosulfan élimine les Miridae qui pourraient se trouver emprisonnés. Une vingtaine de jours après, l'infestation est réalisée dans 3 cages sur 5 , les témoins sont constitués par 2 cages sans punaise. Cette contamination est renouvelée dans la semaine qui suit. En moyenne ces 2 infestations qui concernent tant des larves âgées que des stades imaginaux d'A. lineolatus et L. rugulipennis fournissent un inoculum de 1 punaise pour
7 à 9 gousses différenciées sous le manchon. En 1976, l'irrégularité de la nouaison a impliqué des variations substantielles d'effectifs de punaises introduits par cage pour satisfaire cette condition. En 1977, la nouaison étant homogène on a fait des infestations de même niveau dans chaque cage (60 à 75 punaises). Juste avant l'introduction des Miridae, on marque au moyen d'un fil coloré le maximum de grappes fructifères présentant des stades bien différenciés de gousses, c'est-à-dire d'une part, le stade "gousse verte non enroulée " et d'autre part, le stade « gousse verte enroulée ». En 1976, en raison de la précocité exceptionnelle de maturation liée à la sécheresse, on a de plus marqué également des inflorescences du stade «gousse brune ». A la maturité des gousses, qui intervient à peu près un mois après les infestations, on recueille les inflorescences par lot correspondant aux diverses marques colorées. Dans chaque lot on sépare les gousses de la grappe. C'est dans ces lots que l'on va tirer au hasard les gousses à examiner. Cet examen se fait sous la loupe binoculaire. En ouvrant les gousses au moyen de pinces fines, on classe les graines dans les 3 catégories « saine », « ridée » et «vidée ».

\section{RÉSULTATS}

\section{Eléments acquis dans les champs d'enquête}

\section{a) Climat et analyses hydriques}

La figure 1 nous montre à titre d'exemple les variations de l'humidité du sol d'une parcelle, suivant les niveaux et les dates de prélèvement. On sait que le développement racinaire de la luzerne lui permet de puiser ses réserves sur une grande profondeur. Par ailleurs, sur l'ensemble des mesures réalisées tant en 1974 et 1975 , on ne constate pas de déficit hydrique notable lors des 2 premières dates de

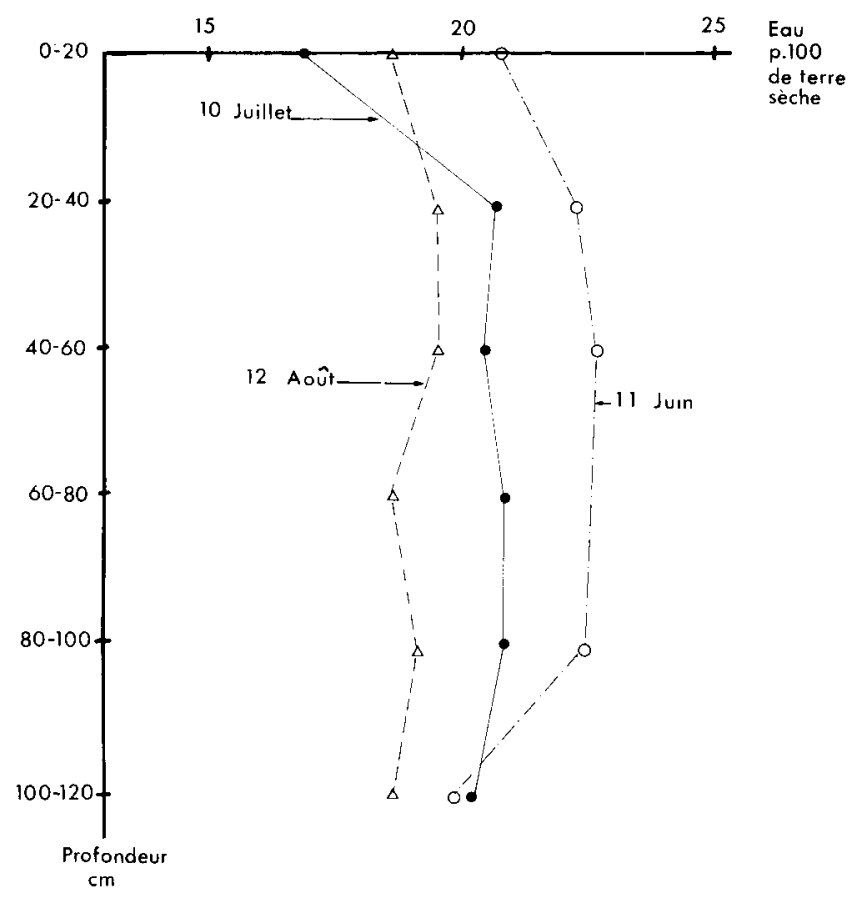

Figure 1

Humidité p. 100 du sol d'un champ d'enquête ( $n^{\circ} 7$ du tabl. 2, 1974) à 3 dates : 11/6 (bourgeonnement), $10 / 7$ (floraison), 12/8 (formation des gousses).

Soil humidity (by weight) of a survey field $\left(n^{\circ} 7\right.$, tabl. 2, 1974) on $11 / 6$ (budding), $10 / 7$ (flowering), 12/8 (pod set). 

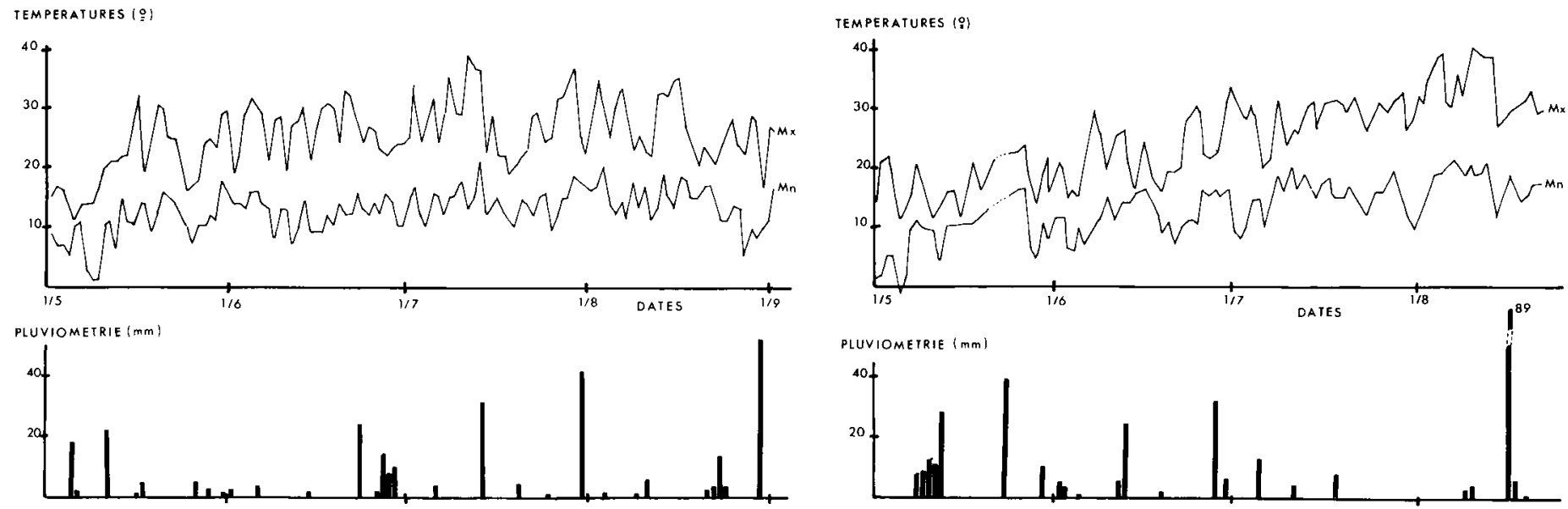

Figure 2

Relevés climatiques de la zone des champs d'enquête (Lavaur, Tarn).

Meteorological data for the area of the survey fields (Lavaur, Tarn).

TABLEAU 2

Résultats des prélèvements effectués lors des 2 années d'enquête dans des parcelles de luzerne porte-graines du Tarn. Results of sampling in the two-year survey of seed-crops in Tarn.

\section{ANNEE 1974}

\begin{tabular}{|c|c|c|c|c|c|c|c|c|c|c|c|c|}
\hline $\begin{array}{l}\text { Champ } \\
\text { Field }\end{array}$ & 1 & 2 & 3 & 4 & 5 & 6 & 7 & 8 & 9 & 10 & 11 & 12 \\
\hline $\begin{array}{l}\text { Note Miridae } \\
\text { Miridae Notation }\end{array}$ & 1 & 2 & 1 & 1 & 3 & 5 & 2 & 5 & 2 & 0 & 5 & 5 \\
\hline $\begin{array}{l}\text { p. } 100 \text { de graines vidées et ridées } \\
\% \text { of shrivelled and emptied } \\
\text { seeds }\end{array}$ & 8,0 & 7,6 & 22,0 & 7,5 & 25,1 & 27,6 & 15,5 & 10,2 & 16,7 & 10,1 & 15,8 & 38,2 \\
\hline $\begin{array}{l}\text { Réserves hydriques le } \\
12 \text { août }(1) \\
\text { Soil ponderal humidity on } \\
\text { Aug. } 12\end{array}$ & $+3,17$ & $-0,59$ & $+3,17$ & $+3,17$ & $-3,11$ & $-0,59$ & $+2,98$ & $-3,22$ & $-1,33$ & $-0,1$ & $-1,33$ & $-1,47$ \\
\hline
\end{tabular}

\section{ANNÉE 1975}

\begin{tabular}{|c|c|c|c|c|c|c|c|c|c|c|c|c|}
\hline $\begin{array}{l}\text { Champ } \\
\text { Field }\end{array}$ & 4 & 5 & 6 & 8 & 9 & 11 & 12 & 13 & 14 & 15 & 16 & 17 \\
\hline $\begin{array}{l}\text { Note Miridae } \\
\text { Miridae Notation }\end{array}$ & 1 & 3 & 5 & 2 & 2 & 2 & 2 & 1 & 2 & 2 & 5 & 2 \\
\hline $\begin{array}{l}\text { p. } 100 \text { de graines vidées et ridées } \\
\% \text { of shrivelled and emptied } \\
\text { seeds }\end{array}$ & 19,9 & 15,4 & 24,4 & 7,7 & 20,1 & 25,7 & 23,5 & 22,7 & 23,3 & 17,7 & 17,4 & 25,4 \\
\hline
\end{tabular}

Réserves hydriques le

12 août (1)

Soil ponderal humidity on

Aug. 7

(1) Ecarts (+ ou -) des humidités (p. 100) à l'humidité au point de flétrissement temporaire.

Differences $(+$ or - ) of humidity $(\%)$ with humidity at wilting point.

prélèvement. On a donc considéré que c'était la dernière date de mesure de l'humidité du sol (vers le 10 août) qui estimait le manque d'eau. A ce moment, on a calculé la moyenne des humidités du sol mesurées aux différents niveaux, afin de ne retenir par parcelle qu'une mesure appréciant les déficits hydriques. Ces derniers sont 
d'ailleurs exprimés dans le tableau 2 par la différence des humidités moyennes à l'humidité au point de flétrissement temporaire.

Les conditions climatiques et notamment la pluviométrie (fig. 2) prouvent le caractère très différent des 2 années de l'enquête: en 1974, de nombreuses précipitations, parfois assez abondantes (plus de $20 \mathrm{~mm}$ par jour) ont eu lieu tout au long de l'été. Les températures ont été irrégulières, à des journées chaudes succédaient des journées froides dues en partie aux précipitations. En 1975, après un printemps et un début d'été très frais d'avril à juin, entraînant un blocage de la pousse de la luzerne, la chaleur a sévi en juillet et août. Des pluies assez régulièrement espacées de mai à début juillet font place, jusqu'à la mi-août à une longue période de sécheresse se terminant par des orages extrêmement violents mais à caractère local, impliquant selon les champs un abat d'eau variant de 0 à $89 \mathrm{~mm}$. Les déficits hydriques en phase de maturation de graines ont donc avant tout marqué l'année 1975. Ils sont exprimés dans le tableau 2.

\section{b) Prélèvement d'insectes et malformation des graines}

La figure 3 montre, à titre d'exemple pour un champ, les variations d'effectifs des Hétéroptères. Lors de la phase de maturation, l'abondance des punaises dans ce champ a atteint la note 5 . Les contaminations lors des 2 annécs sont

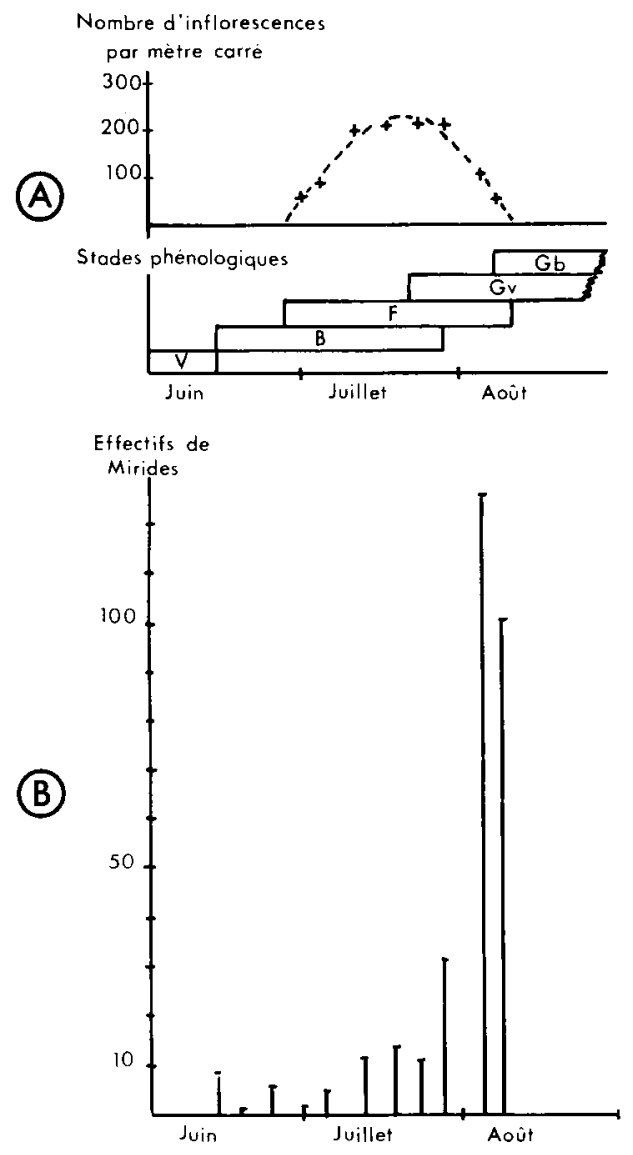

Figure 3

Phénologie de la luzerne (A) et nombre de punaises Mirides (B) capturées en 25 coups de filet d'un champ d'enquête ( $n^{\circ} 8$ du tabl. 2 , 1974).

Lucerne phenology (A) and number of Mirid bugs $(B)$ in 25 sweepnet samples in a survey field $\left(n^{\circ} 8\right.$, tabl. 2,1974$)$.

$V=$ végétatif; vegetative.

$B=$ bourgeonnement $;$ budding

$F=$ floraison ; flowering.

$G v=$ gousses vertes; green pods.

$G b=$ gousses brunes; brown pods. comparables puisque les moyennes des notes suivies de leur intervalle de confiance sont de $2,7 \pm 1,2$, en 1974 , et $2,4 \pm 0,8$, en 1975 . Les pourcentages moyens de malformation des graines sont constants lors des 2 années d'enquête puisque l'on trouve respectivement pour les graines vidées 8,2 p. 100 et 9,0 p. 100 en 1974 et 1975 , puis 10,1 et 11,1 p. 100 pour les graines ridées. Les écarts à ces moyennes sont cependant beaucoup plus marqués en 1974 puisqu'il y a 5 champs sur 12 qui se situent de 5 à 10 p. 100 de graines malformées alors qu'en 1975 il n'y a qu'une seule parcelle qui soit dans ce cas. Certains champs ont donc plus particulièrement souffert de la sécheresse estivale, en 1975. Dans les 7 champs qui ont été suivis durant les 2 années d'enquête, il n'y a pas de corrélation significative soit entre les populations annuelles de punaises, soit entre les déficits hydriques annuels.

\section{Eléments acquis dans les essais d'infestation artificielle}

Les 2 années où les essais ont été pratiqués sont très différentes sur le plan du déficit hydrique climatique (fig. 4) qui ne tient pas compte des réserves du sol. En 1976, année particulièrement sèche, avant la première irrigation ( 22 juillet) les réserves hydriques du sol étaient déjà fortement entamées, comme le prouve le dessèchement sensible jusqu'à $1 \mathrm{~m}$ de profondeur mais non épuisées (fig. 5). Les

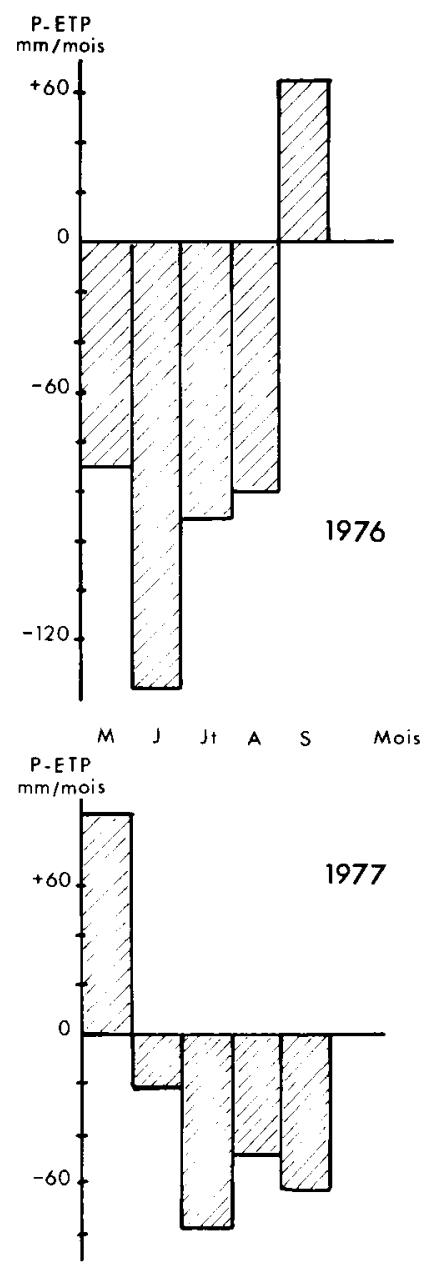

Figure 4

Déficits hydriques climatiques à Lusignan (1976 et 1977).

Climatic water deficit at Lusignan (1976 and 1977).

$P=$ précipitations $=$ rainfall.

ETP = evapotranspiration potentielle PENMAN

P.E.T. = potential evapotranspiration PENMAN. 
Figure 5

traits pointillés : Bilan des réserves hydriques du sol à Lusignan (1976) dans les parcelles expérimentales à deux dates.

dotted lines: soil humidity (by volume) at Lusignan (1976) in experimental plots on two dates.

non $I=$ non irrigué $=$ no irrigation.

$I=$ irrigué $=$ irrigated .

traits pleins : full lines.

* capacité au champ : field capacity.

* P.F. $4,2=$ point de flétrissement permanent $=$ wilting point

N.B. : Humidité volumique $=$ Humidité pondérale $\times$ densité apparente.

Humidity (by volume) $=$ humidity (by weight) $\times$ apparent density.

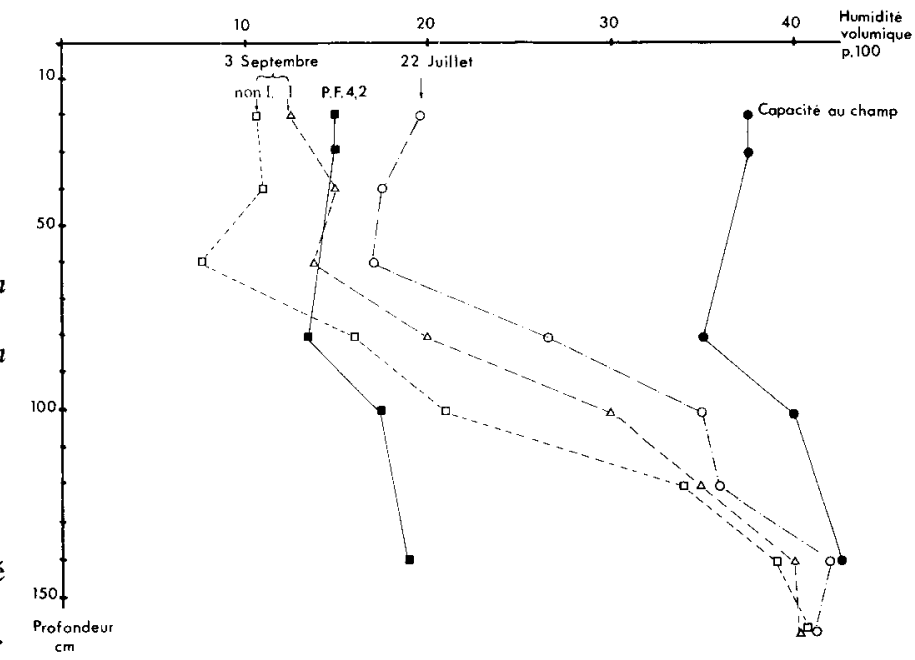

TABLEAU 3

Répartition ( $p .100)$ des catégories de graines à la récolte dans les essais d'infestation par les Miridae dans les parcelles irriguées et non irriguées. Distribution of seed categories (\%) observed at harvest in the infestation experiments with Miridae, with or without irrigation.

\begin{tabular}{|c|c|c|c|c|c|c|c|c|}
\hline \multirow{3}{*}{$\begin{array}{l}\text { Stade des lots de gousses marqués } \\
\text { lors de l'infestation par les Miridae } \\
\text { Pod phenology at the moment } \\
\text { of the introduction of Miridae }\end{array}$} & \multicolumn{8}{|c|}{$\begin{array}{c}\text { 1. Essais } 1976 \\
\text { 1976 Experimentations }\end{array}$} \\
\hline & \multicolumn{4}{|c|}{$\begin{array}{l}\text { Parcelle irriguée } \\
\text { irrigated plot } \\
\text { p. } 100 \text { de graines } \\
\% \text { of seeds }\end{array}$} & \multicolumn{4}{|c|}{$\begin{array}{l}\text { Parcelle non irriguée } \\
\text { unirrigated plot } \\
\text { p. } 100 \text { de graines } \\
\% \text { of seeds }\end{array}$} \\
\hline & $\begin{array}{l}\text { saines } \\
\text { sound }\end{array}$ & $\begin{array}{c}\text { ridées } \\
\text { shrivelled }\end{array}$ & $\begin{array}{l}\text { vidées } \\
\text { emptied }\end{array}$ & $\begin{array}{l}\text { test } \\
\times 2\end{array}$ & $\begin{array}{l}\text { saines } \\
\text { sound }\end{array}$ & $\begin{array}{l}\text { ridées } \\
\text { shrivelled }\end{array}$ & $\begin{array}{l}\text { vidées } \\
\text { emptied }\end{array}$ & $\begin{array}{l}\text { test } \\
\times 2\end{array}$ \\
\hline $\begin{array}{l}\text { Gousse verte non enroulée } \\
\text { unrolled green pod } \\
\text { - infesté } \\
\text { infested } \\
\text { - témoin } \\
\text { control }\end{array}$ & $\begin{array}{l}71,3 \\
87,0\end{array}$ & $\begin{array}{r}17,1 \\
2,0\end{array}$ & $\begin{array}{l}11,5 \\
11,0\end{array}$ & ths & $\begin{array}{l}53,1 \\
75,1\end{array}$ & $\begin{array}{r}29,4 \\
0,7\end{array}$ & $\begin{array}{l}17,5 \\
24,2\end{array}$ & ths \\
\hline $\begin{array}{l}\text { Gousse verte enroulée } \\
\text { rolled green pod } \\
\text { - infesté } \\
\text { infested } \\
\text { - témoin } \\
\text { control }\end{array}$ & $\begin{array}{l}68,5 \\
66,3\end{array}$ & $\begin{array}{l}11,7 \\
11,3\end{array}$ & $\begin{array}{l}19,8 \\
22,4\end{array}$ & ns & $\begin{array}{l}55,7 \\
70,4\end{array}$ & $\begin{array}{l}19,0 \\
11,9\end{array}$ & $\begin{array}{l}25,3 \\
17,7\end{array}$ & ths \\
\hline $\begin{array}{l}\text { Gousse brune } \\
\text { brown pod } \\
\text { - infesté } \\
\text { infested } \\
\text { - témoin } \\
\text { control }\end{array}$ & $\begin{array}{l}34,7 \\
51,4\end{array}$ & $\begin{array}{l}48,6 \\
26,8\end{array}$ & $\begin{array}{l}16,7 \\
21,8\end{array}$ & ths & $\begin{array}{l}12,1 \\
24,3\end{array}$ & $\begin{array}{l}61,4 \\
57,3\end{array}$ & $\begin{array}{l}26,5 \\
18,4\end{array}$ & ths \\
\hline
\end{tabular}

Gousse verte non enroulée

unrolled green pod

- infesté

infested

- témoin

control

2. Essais 1977

1977 Experimentation

Gousse verte enroulée

rolled green pod

- infesté

infested

- témoin

control

$\begin{array}{rrrrrrrr}76,9 & 6,0 & 17,1 & \text { ths } & 73,9 & 14,6 & 11,5 & \text { ths (1) } \\ 88,1 & 6,3 & 5,7 & & 76,7 & 7,4 & 15,9 & \end{array}$

(1) ns si l'on groupe les graines ridées et vidées, voir texte. ns when pooling shrivelled and emptied seeds. 
2 irrigations ( $100 \mathrm{~mm}$ en tout) apportées jusqu'au 3 septembre n'ont fait que maintenir l'état hydrique du sol au voisinage du point de flétrissement dans les 60 premiers $\mathrm{cm}$, ne permettant à la luzerne de s'alimenter facilement en eau qu'au-delà de cette profondeur. Compte tenu que nous nous trouvions en année d'installation de la culture, l'exploitation des couches profondes du sol n'était pas bien assurée et de ce fait, nous n'avons pas observé de dessèchement du sol audelà de 1,20 m. L'irrigation n'a pas pu, en 1976, maintenir la luzerne dans les conditions d'alimentation hydrique optimales. Cependant, les parcelles sèches se sont trouvées dans des conditions encore plus défavorables ce qui s'est traduit par une consommation déficitaire de $50 \mathrm{~mm}$ par rapport à celle des parcelles irriguées dans la période de maturation des graines (22 juillet au 30 septembre). En 1977, l'irrigation a débuté le 21 juillet. L'année relativement humide n'a pas permis de mettre en évidence des différences de consommation d'eau entre les 2 types de parcelles.

On soulignera d'ailleurs que la sécheresse estivale de 1976 est à l'origine d'une part très importante des graines malformées dans les gousses brunes. Dans ce cas comme pour les gousses moins évoluées, les punaises accentuent la gravité des dégâts. Cela ressort des fréquentes significations du test $\chi_{2}$ appliqué aux effectifs de graines. Dans le lot "gousse verte non enroulée » de la parcelle non irriguée 1977, on constate peu de différences entre les pourcentages de graines saines du lot témoin et du lot infesté. En fait, si on rassemble l'ensemble des graines vidées et ridées en une seule catégorie "graines malformées", l'effet des punaises devient non significatif. C'est le seul cas où une telle modification doit être mentionnée.

\section{DISCUSSION ET CONCLUSIONS}

En 1974 et 1975, comme lors d'enquêtes précédentes, on a noté des effectifs élevés d'Hétéroptères Miridae dans le Tarn. La précocité des cultures dans cette zone du Sud de la France implique que les dégâts qu'occasionnent ces punaises concernent la phase de maturation des graines et très rarement les bourgeons ou les fleurs. On peut donc établir la corrélation entre les malformations de graines et l'abondance des punaises. En 1974, on trouve un coefficient de corrélation $\mathrm{r}=+0,54$, qui est proche de la signification $(0,05<\alpha<0,10$ pour 10 d.d.l.). Cependant la connaissance des déficits hydriques nous permet, lors de la $1^{\text {re }}$ année d'enquête, d'éliminer de la relation les parcelles 5 et 8 où le manque d'humidité relative du sol est net. Dans ce cas la corrélation s'établit à $r=+0,70$ (pour 8 d.d.1.). En 1975 en revanche, une analyse analogue ne conduit jamais à une relation significative. En fait, lors de la $2^{\mathrm{e}}$ année, les déficits hydriques ont joué un rôle prépondérant mais la date du dernier contrôle d'humidité est sans doute trop précoce pour permettre de saisir complètement le déficit en phase de maturation des graines.

Les expérimentations réalisées en infestation artificielle nous permettent de souligner ce rôle cumulatif de l'eau et des punaises sur l'état des graines. Ainsi, en 1976, année particulièrement sèche, dans les 3 stades repères de gousses, on trouve que les malformations du lot infesté en conditions irriguées sont sensiblement comparables au lot témoin en conditions non irriguées. Dans le cas de la zone irriguée, les punaises accroissent jusqu'à 15 p. 100 les malformations. Ce pourcentage se situe à plus de $20 \mathrm{p} .100$ pour la zone non irriguée. Les résultats de 1977 sont moins nets.

Si dans la zone irriguée les malformations sont accrues de $10 \mathrm{p} .100$, il n'y a pas de rôle très évident des punaises dans le lot non irrigué sans qu'on puisse y apporter une explication expérimentale. Cependant il faut souligner que nous n'avons retenu pour notre interprétation que le critère qualité de la graine alors que l'action des Miridae joue également sur les chutes de gousses donc sur le nombre total de graines à la récolte (BouRnoville, 1975).

L'ensemble de nos résultats précise des éléments publiés dès 1936 par SoRENSON. Cet auteur soulignait que les Lygus $s p$. occasionnaient sur la luzerne porte-graines un accroissement des graines malformées. Il constatait cependant que ce type de semences existait également dans les cages «témoins» ce qui le faisait conclure à l'existence d'autres causes de ces dégâts. Malgré la relative abondance de la littérature consacrée à l'action déprédatrice des Mirides sur la production de graines de luzerne depuis cette date, on ne retrouve guère que dans SEDIVY (1972) l'idée qu'une luzernière souffrant d'un manque d'alimentation en eau a plus de difficultés à supporter l'action des Miridae. Il est vrai que les symptômes de piqûres des Miridae sur les divers stades de la luzerne manquent, alors que divers auteurs les ont rapportés sur d'autres cultures comme le blé (NuORTEVA, 1956) ou le cotonnier (MAUney \& HenNEBERRY, 1979).

On peut néanmoins prendre l'exemple des travaux réalisés sur la lutte intégrée contre les Lygus sur cette dernière culture pour souligner l'importance des facteurs qui agissent avec les insectes pour l'expression des pertes de rendement d'un végétal.

Reçu le 8 juillet 1981. Accepté le 20 octobre 1981

\section{REMERCIEMENTS}

Nous exprimons nos remerciements à M. LEMAIRE (I.N.R.A. Agronomie, Lusignan) pour nous avoir fourni l'analyse critique des résultats des réserves hydriques du sol de l'essai de Lusignan. Nous sommes également redevables à nos collègues des Stations d'Agronomic de Toulouse et de Lusignan d'avoir réalisé les analyses des déficits hydriques.

\section{RÉFÉRENCES BIBLIOGRAPHIQUES}

Bournoville R., 1975. Relations entre les Hétéroptères Mirides nuisibles à la luzerne porte-graines et la phénologie de la plante. Ann. Zool. Ecol. anim., 7, 197-210.

Bournoville R., 1976. Méthodes d'étude des dégâts occasionnés par les insectes séminivores de la luzerne. Rev. Zool. agric. Pathol. vég., 75, 56-66.

Bournoville R., Bourdoncle B., 1978. Les populations d'insectes ravageurs de la luzerne porte-graines dans le Tarn. Leur action sur le rendement grainier. Publication - I.N.R.A. Pont de la Maye, $30 \mathrm{p}$.

Bournoville R., Delaude A., 1975. Résultats d'enquêtes sur les insectes phyllophages et séminivores de la luzerne. Rev. Zool. agric. Pathol. vég., 74, 90-107.

Mauney J. R., Henneberry T. J., 1979. Identification of damage symptoms and patterns of feeding of plant bugs in cotton. J. econ. Entomol., 496-501.

Nuorteva P., 1956. Studies on the salivary secretions of some Heteroptera and Homoptera on plant growth. Ann. Entomol. fenn., 22, 108-117.

Peyremorte P., 1974. L'alimentation en eau des cultures de luzerne destinées à la production de graines. Bull. inf. FNAMS, 12, 37-41.

Rotrekl J., 1973. Investigation concerned to noxiousness caused by the lucerne plant bug (Adelphocoris lineolatus Goeze) on lucerne seed stands. Sb. vedeckych Praci, 3, 161-173.

Sedivy J., 1972. Dic Schädlichkeit der Wanze Lygus rugulipennis Popp. an Samenluzerne. Z. Pflanzenkr. Pflanzenschutz, 79, 7, 407412.

Sorenson C. J., 1936. Lygus bugs in relation to occurrence of shrivelled alfalfa seeds. J. econ. Entomol, 29, 454-457. 